\title{
Primarias abiertas presidenciales en el Cono Sur ¿un método necesario y conveniente? Análisis de los casos de Argentina, Chile y Uruguay
} Ariadna Gallo

\author{
Universidad Nacional de San Martín
}

\section{Resumen}

En este trabajo se indaga acerca del sistema de internas o primarias abiertas, en los tres países - Argentina, Chile y Uruguay- en los que éstas fueron formalmente adoptadas, a través de una perspectiva metodológica comparada. Aquí procuraremos averiguar si el procedimiento de las primarias abiertas es necesario (es decir, si los beneficios perseguidos con su aplicación emergen como respuestas a problemáticas existentes en la sociedad) y si es conveniente (si se producen determinadas condiciones que permitan que tales beneficios superen los costos ocultos que acarrea), evaluando todas las elecciones transcurridas desde su incorporación. Observamos que los beneficios buscados no se han obtenido hasta ahora, en ninguno de los tres casos; lo cual terminó promoviendo la fragmentación, la inconsistencia interna y la pérdida de relevancia de los partidos políticos en el marco de un sistema democrático.

Palabras clave: Primarias abiertas, Regulación oficial, Argentina, Chile, Uruguay, Partidos políticos.

Open presidential primaries in the Southern Cone: a necessary and convenient method? Analysis of the cases of Argentina, Chile and Uruguay

\begin{abstract}
This paper will evaluate the effect of the open primary elections system, in the three countries -Argentina, Chile and Uruguay- in which this institutional engineering instrument was formally adopted and regulated by the electoral organism of the country. We will try to find out if the open primary procedure is necessary (if the benefits sought by its application emerge as responses to existing problems in society) and whether it is appropriate (if certain conditions exist that allow such benefits to outweigh the hidden costs that it entails), evaluating its functioning in every election since its incorporation, and comparing the results to ascertain the evolution of the implementation of this norm. We note that these advantages have not been obtained so far, in any of the three cases (particularly in Argentina, where the party system is much weaker and less institutionalized than in those of its counterparts). The use of this system resulted in an improvement of the political parties in none of these cases, giving rise instead to the establishment of a type of nonpartisan, personalized or divided competition in sectors. This ended up promoting fragmentation, internal inconsistency and the loss of relevance of political parties within the framework of a democratic system.
\end{abstract}

Keywords: Simultaneous open primaries, Official regulation, Argentina, Chile, Uruguay, Political parties.

E-mail: doctoraag75@hotmail.com 


\section{Introducción}

En las reformas político-institucionales llevadas a cabo durante las últimas décadas en América Latina, una de las dimensiones privilegiadas ha sido aquella concerniente a la organización intrapartidaria y a los mecanismos de nominación de candidaturas partidarias (Freidenberg, 2016) la mayor parte de las propuestas de reforma política han girado en torno a la incorporación de alguna variante del mecanismo de primarias o internas abiertas presidenciales -que consiste en que la nominación de candidaturas partidarias sea realizada a través de una elección en la que se autoriza a participar a todo ciudadano empadronado en el registro electoral nacional del país (Gallagher y Marsh, 1988; Freidenberg, 2003). A pesar de ello, son pocos los países del subcontinente latinoamericano en los que rigen actualmente disposiciones formales que impongan la realización de internas abiertas a nivel presidencial.

En este trabajo nos concentraremos en el estudio de los casos de Argentina, Chile y Uruguay, los únicos tres países latinoamericanos que han incluido en su ordenamiento jurídico el sistema de elecciones primarias abiertas mediante el voto universal, directo y secreto, con participación de todos los electores para la selección de las candidaturas y las agrupaciones políticas, convocadas y controladas por las autoridades estatales. Y que, a su vez, se llevaron a cabo en más de una oportunidad.

Aquí se pasará revista por los argumentos que han respaldado la incorporación de este mecanismo, diferenciando explícitamente la justificación teórica proporcionada por sus propulsores (basada generalmente en la literatura teórica existente en la materia), de las motivaciones políticas reales de cada coalición de gobierno que impulsó la reforma en la legislación electoral.

Por empezar, los fundamentos teóricos que sustentan la incorporación del procedimiento de primarias abiertas, por lo general, apuntan a las siguientes ideas: otorgar mayor protagonismo ciudadano en la vida partidaria; racionalizar y ordenar el sistema partidario; mejorar el funcionamiento interno partidario; legitimar al representante electo; recuperar la confianza de los electores en los partidos; renovar la política y recomponer el vínculo entre representantes y representados (Colomer, 2002; Alcántara Sáez, 2002; Haro, 2002; Alcántara Sáez y Freidenberg, 2003; Muñoz, 2003; Carey y PolgaHecimovich, 2006).

Con respecto a las motivaciones políticas reales de los impulsores del cambio de normativa de selección interna, hay que atender a las particulari- dades de la coyuntura política en cada uno de los tres casos a analizar. En Uruguay, el sistema de internas abiertas surgió con la reforma constitucional de 1997, mediante la cual se sustituyó al doble voto simultáneo y acumulativo (también llamado Ley de Lemas) y se puso en práctica un sistema de tres fases para los comicios presidenciales (internas abiertas partidarias, elecciones generales y, eventualmente, balotaje). Allí, la sustitución del sistema precedente por este dispositivo de tres fases tuvo el objetivo de adaptarse a la alteración en el sistema partidario, sufrida a mediados de los $90^{6} \mathrm{y}$ frenar el crecimiento del FA, incluyendo un mecanismo que permitiera aunar las fuerzas de los partidos mayoritarios para evitar que ese frente llegara al poder.

En Argentina, en el año 2009, se sancionó la Ley de Democratización de la Representación Política, la Transparencia y la Equidad Electoral (Nro. 26.571), mediante la cual se formalizó el método de primarias abiertas simultáneas y obligatorias (PASO) para los cargos de elección popular nacional, siendo éstas organizadas, controladas y tuteladas por el organismo electoral nacional del país. Esta reforma fue propuesta por la coalición gubernamental en un momento en que el oficialismo declinaba en popularidad, procurando con ella reunificar a un peronismo por entonces dividido, en torno a la fracción oficialista y, a la vez, disminuir la capacidad operativa de sus rivales (Caruncho, 2014).

En Chile, a fines de 2009, se incorporó una ley orgánica constitucional (Ley $\mathrm{N}^{\circ} 20.640$ ) que establecía un sistema de elecciones primarias facultativas, es decir, que podría ser utilizado voluntariamente por los partidos políticos para la nominación de candidatos a Presidente de la República, parlamentarios y alcaldes, de carácter nacional, organizadas por el Servicio Electoral (Servel), a celebrarse en una misma fecha para todos los partidos o pactos a la vez (www.servel.cl/). En este país, las primarias abiertas se incorporaron en el medio de un clima político de cambio en el que se reclamaba el fin de la binominalidad y la apertura del sistema, con el objeto de dar una solución a la creciente fraccionalización interna de las dos coaliciones principales.

Aunque las motivaciones políticas concretas fueron diferentes entre ellos, en casi todos los casos la línea argumental seguida coincidía con las justificaciones comúnmente utilizadas en la literatura teórica que aborda el tema ; según las cuales la utilización de primarias abiertas conduciría a la obtención de efectos beneficiosos para los partidos y el sistema de partidos, fortaleciendo los vínculos entre éstos y la ciudadanía común. 
Ahora bien, en esta investigación nos centraremos principalmente en el análisis de los argumentos teóricos que respaldaron la incorporación de este mecanismo (subrayando sus inconsistencias o contradicciones internas); para lo cual pasaremos revista por la literatura especializada en la temática (diseccionando los argumentos y hallazgos obtenidos, organizándolos en función de los principales objetivos teóricos buscados). Luego estudiaremos comparativamente los tres casos mencionados, en todas las oportunidades en las que se utilizó este método para seleccionar a los candidatos presidenciales (1999, 2004, 2009 y 2014 en Uruguay; 2011 y 2015 en Argentina; y 2013 y 2017 en Chile), teniendo en cuenta las diferencias originariamente existentes entre ellos. Si bien son escasas las situaciones analizadas, sostenemos provisoriamente que hasta el momento no ha habido una correlación positiva entre el empleo de este método selectivo y el cumplimiento de los beneficios pautados teóricamente con la aplicación del mismo.

Como se dijo, la incorporación oficial de la normativa de primarias/internas abiertas ha sido el denominador común en los tres casos. Aquí, en primer lugar, consideraremos que, como regla general, existen dos criterios básicos que se utilizan a la hora de incorporar determinada normativa, a saber: necesidad (utilidad distintiva de un procedimiento para resolver un problema de modo que ningún diseño alternativo podría lograr) y conveniencia (que se refiere a neutralización de los costos ocultos generados por la imposición del mismo) (Pérez-Liñán, 2002). Así, diremos que el procedimiento de primarias abiertas es necesario si se trata del mejor mecanismo para obtener ciertos beneficios que, a su vez, emergen como respuestas a problemáticas a enmendar, resolver o reformar, existentes en la sociedad. Mientras que estimaremos que el instrumento es conveniente si se producen determinadas condiciones que permitan que tales beneficios se concreten y que, al mismo tiempo, superen los costos ocultos que la aplicación de este sistema acarrea. Para ello, estableceremos un estándar normativo de evaluación, es decir un umbral de rendimiento deseado esperado con la implementación de las primarias abiertas (Pérez-Liñán, 2002). En segundo término, efectuaremos una evaluación de índole comparativa entre los tres casos, a partir de la cual sondearemos si la técnica incorporada efectivamente permite lograr ciertos resultados y procuraremos establecer si se cumplen adecuadamente determinadas condiciones para lograr el estándar normativo estipulado.

\section{Estado de la cuestión: sistema de internas/primarias abiertas y simultáneas}

Con el sistema de primarias reguladas por ley se busca que la selección de los candidatos sea efectuada por un cuerpo mayoritario de individuos en lugar de recaer en el líder o en un sector minoritario a través de métodos orgánicos partidistas (Tullio, 2011). Las primarias abiertas constituyen un peculiar mecanismo de selección de candidaturas (MSC), estipulado para nominar al candidato único y oficial del partido o coalición partidaria (Colomer, 2004: 96); sin embargo, no es el único mecanismo democrático, ni siquiera es el más efectivo (Scarrow et al., 2000; Kittilson y Scarrow, 2003). Existen distintos tipos de MSC, que pueden dividirse entre "democráticos" y "no democráticos" (Colomer, 2002; Muñoz, 2003), en función de su selectorado - es decir, del cuerpo que selecciona al candidato (Rahat y Hazan, 2001). Las variantes comprenderían: la designación por parte de líder o élite partidaria (no democrático); la elección por congreso o convención (puede ser democrático si los delegados son elegidos por las bases, o no democráticos si son nominados por la élite); las primarias/internas cerradas de afiliados o militantes (democrático) y las primarias abiertas al electorado total de un país (democrático) (Cantillana Peña et al., 2015; Muñoz, 2003). Estas opciones se ordenan en función de su nivel de inclusividad creciente, pero también del grado de homogeneidad decreciente del selectorado.

En efecto, con primarias abiertas, el selectorado está compuesto (al menos potencialmente) por el electorado general, el cual se subdivide básicamente en dos grandes sectores: el activo (conformado por los "core supporters" o militantes y adherentes férreos de los partidos existentes, que actualmente constituyen una porción muy minoritaria dentro del conjunto de votantes totales) y el pasivo (compuesto por "swing voters", electores sin preferencias que suelen predominar ampliamente en el conjunto del electorado general) (Ting et al., 2018). Justamente, el objetivo de la incorporación de primarias obligatorias por ley era generar incentivos directos a la participación ciudadana, con la finalidad de licuar las diferencias entre el segmento activo y el segmento pasivo del electorado. 
Tabla 1: Beneficios de las primarias abiertas, según áreas mencionadas

ÁREA

Sistema de partidos; Criterio para

elaborar preferencias ciudadanas
BENEFICIOS

Ordenamiento del sistema partidario

Reducción de partidos y candidatos Mayor potencial para coaligarse

Tipo de partidos y sus funciones; Elección de candi- Democratización

datos

Vínculos entre dirigentes y partidos

Viabilidad (de partidos y candidatos) Legitimidad

Participación

Vínculo entre ciudadano y partido

Manifestación de preferencias ciudadanas

Acercamiento de la ciudadanía a los partidos

\section{Objetivos teóricos buscados con el sis- tema de primarias abiertas}

Como se vio, en la literatura teórica que respalda la incorporación del mecanismo de primarias abiertas, habitualmente se subraya que a través de las mismas pueden lograrse los siguientes beneficios:

- Ordenamiento de la oferta y de la dinámica partidaria. Se sostiene que este sistema facilita la presencia de organizaciones fuertes, duraderas y con significativo anclaje social (Tullio, 2011). En efecto, las internas/primarias abiertas como regla electoral pueden producir efectos mecánicos (eliminación de actores que no superen el piso o umbral establecido) y también psicológicos (conformación de alianzas entre fuerzas afines para poder competir en ellas) (Duverger, 1954; Cox, 2004). En Argentina, el establecimiento de pisos o umbrales para acceder a la elección general, tenía como objeto, por un lado, reducir el número de agrupaciones que competían (Escolar, 2011), mitigando la dispersión existente y, por otro, promover la unidad entre partidos similares (Abal Medina, 2009; Astarita, 2009).

- Democratización interna partidaria. Se argumenta que las primarias contribuyen a reducir el poder del aparato partidario (Alcántara Sáez, 2002). Según se ha expuesto, con las internas abiertas se desapodera a las cúpulas de ciertas funciones (Blanco Valdés, 1996; Hernández Valle, 2002), se restringe el espacio de las oligarquías partidarias (Lawson, 1994) $\mathrm{y}$, en consecuencia, se favorece el ejercicio de los derechos de los miembros de los partidos (Vargas Machuca, 1998), abriendo diques a la participación ciudadana (Auth, 2005).

- Legitimidad añadida hacia el ganador. A su vez, las primarias permiten "descubrir" a un "tapado" (Colomer, 2002; Cantillana Peña et al., 2015), a un party adherent (Siavelis y Morgenstern, 2008) o a un dirigente no apoyado por el aparato partidario, quien no obstante podría representar adecuadamente al partido en la elección general. O alternativamente pueden utilizarse para establecer la candidatura en una coalición preexistente, con sectores internos con un peso equivalente, resolviendo la correlación intestina de fuerzas al interior de aquella (Navia y Rojas Morales, 2008).

- Viabilidad al candidato triunfador y a la etiqueta partidaria. Se dice que este mecanismo otorga valiosa información a los ciudadanos respecto de cómo se distribuyen las preferencias de los otros votantes y de la probabilidad de triunfo de las alternativas en competencia (Meirowitz, 2005; Tullio, 2011González y Vargas, 2013; Ernesto y Pomares, 2015). De este modo, se produciría una clarificación respecto de las expectativas de viabilidad de los contendientes calificados (Reynoso, 2015). Al mismo tiempo, le permitiría al elector premiar al partido que hubiera optado por la utilización de este mecanismo de selección de candidaturas (Carey y Polga-Hecimovich, 2006).

- Incremento de la participación y de las instancias de manifestación de preferencias ciudadanas, lo que implica otorgarle al votante la oportunidad de sufragar dentro de su partido o frente favorito (o dentro de alguno de aquellos entre los cuales está indeciso), eligiendo a su candidato predilecto de entre los que se presentan, postergando para la instancia siguiente, el señalamiento estratégico hacia el postulante más aceptable dentro del conjunto de opciones viables (Cox, 2004). Es decir, este sistema no ha sido diseñado para que el elector elija a su candidato preferido en abstracto, sino para que efectúe una preselección dentro de una estructura o espacio predeterminado (un partido constituido o una coalición pluripartidaria). Esto presupone que los ciudadanos experi- 
mentan una identificación con aquellas fuerzas que compiten . A su vez, cuanto menos intensa sea la identificación de los ciudadanos con las formaciones políticas existentes, más probabilidad de que no concurran voluntariamente a votar y cuanto más difusa sea la dirección de la identificación, menos plausible será que emitan un voto sincero.

Ahora bien, una vez identificados los beneficios que teóricamente podría proporcionar la utilización de este mecanismo, estableceremos -también a partir de la literatura clásica y actual y de los hallazgos obtenidos en trabajos recientes- ciertas condiciones necesarias para alcanzar un determinado estándar normativo en el cumplimiento de tales beneficios.

Las condiciones estipuladas como necesarias para alcanzar un estándar normativo aceptable provienen de hallazgos obtenidos en estudios que, en su mayoría, se centran en primarias unilaterales (es decir, sin regulación oficial). Sin embargo, son aplicables a casos de un sistema regulado, habiendo sido adaptadas a las especificidades de estos casos: por ejemplo, la noción de primary bonus y la idea de voto sincero, direccionado e intenso (que en este caso se ligan a la simultaneidad y a la obligatoriedad de la participación ciudadana en las primarias) .

De todos modos, cabe señalar que varias de las condiciones señaladas en la tabla entran en contradicción entre sí ; con lo cual se torna poco probable la obtención de todos los beneficios esperados con este mecanismo de nominación de candidaturas. A todo esto hay que agregarle que las elecciones primarias conllevan un incremento de los costos del proceso y un desgaste generado por la doble campaña (Page, 2016) y la confrontación pública entre candidatos de un mismo espacio político (Sabag, en González y Vargas, 2013; Altman, 2012; Agranov, 2011).

Ahora bien, la hipótesis que guiará este trabajo es que con la aplicación del sistema de internas o primarias abiertas no suelen lograrse los objetivos esgrimidos por los promotores del mismo (ordenamiento del sistema partidario; democratización interna; viabilidad de los partidos y candidatos; legitimidad; participación y manifestación de preferencias de los ciudadanos), en la medida en que no es frecuente que se cumplan todas las condiciones estipuladas (reducción de oferta; criterios de ordenamiento de largo plazo; sustitución de un MSC no democrático; competencia; competitividad; ausencia de conflictividad; obtención de bonus; batacazo; doble legitimidad; retención coalicional; voto direccionado y sincero y manifestación de preferencia intensa) que permiten alcanzar un determinado estándar normativo que posibilite la consecución de aquellos beneficios. A su vez, consideramos que este sistema no resulta necesario, porque en la mayor parte de los casos no constituye el mejor mecanismo para lograr las mejoras requeridas en áreas específicas en las que se presentan problemáticas, ni tampoco conveniente, ya que por lo general los costos ocultos superan los potenciales beneficios. Todo esto se agrava en sistemas en los cuales los partidos previamente son débiles y los ciudadanos no se sienten identificados con éstos.

\section{Variables, dimensiones e indica- dores}

Para el análisis de los casos, utilizaremos como variables los elementos que los defensores de las internas/primarias abiertas conciben como los objetivos buscados con las mismas, desarrolladas precedentemente.

\section{Los tres casos analizados}

Cabe señalar que cualquier propuesta de reforma tiene que estar precedida por un diagnóstico apropiado, en el que primero se identifique el/los problema/s, luego se describan sus características y posteriormente se proponga una posible solución. Por ello, aquí empezaremos exponiendo cómo era la situación de previa a la incorporación de la ley en cada uno de los casos, exhibiendo potenciales problemáticas a enmendar, para poder posteriormente interrogarnos acerca de la incorporación de primarias abiertas como mecanismo más efectivo para la resolución de las mismas.

En esta tabla se puede observar que la situación de partida era diferente en cada uno de los tres casos y que las problemáticas que se buscó enmendar con el sistema de primarias abiertas no eran comunes a todos ellos.

\section{Las internas o primarias abiertas en cada uno de los casos}

A continuación veremos las diversas variantes y disposiciones formales del sistema de primarias abiertas en cada uno de los tres casos analizados:

En lo que sigue, expondremos sintéticamente, cómo se sucedieron las internas abiertas en cada uno de los tres países, en todas las oportunidades en que fue implementada esta metodología hasta la fecha, considerando los ítems anteriormente mencionados. 
Tabla 2: Beneficios de las primarias abiertas y condiciones para lograr estándar normativo Beneficios teóricos Condiciones para su cumplimiento

Ordenamiento del Reducción cuantitativa y cualitativa de la oferta partidaria: que el escenario electoral sistema partidario quede más depurado y ordenado de acuerdo a criterios consistentes y de largo plazo. Criterios de ordenamiento consistentes y de largo plazo. Es decir, que la unidad lograda entre partidos repose sobre cuestiones sólidas, relativas al proyecto político o al posicionamiento ideológico de los miembros coligados.

Democratización Sustitución de un MSC no democrático utilizado previamente; es decir, con un selecinterna partidaria torado minoritario. Esto abarcaría designación por parte de líder o élite partidaria; o elección por congreso o convención, con delegados electos por un cuerpo minoritario. Competencia y competitividad. Para que la selección la realice la ciudadanía, debe haber al menos dos contendientes competitivos y diferenciados.

Contiendas pacíficas. Las pugnas internas conflictivas pueden producir un efecto divisivo que deja secuelas en las organizaciones y promueven defecciones de los perdedores, desincentivando a los dirigentes a seguir militando.

Viabilidad (de Efecto incremental o efecto batacazo. El primero implica la superación de marcas partidos y en elecciones generales. El segundo se refiere a la inesperada performance positiva candidatos) de un partido/sector/candidato en las primarias, que provoca una reconfiguración de expectativas a su favor. Este puede ser interno (si se impone un sector o precandidato inesperado) o general (si dentro del conjunto de partidos, hay uno que resulta sorpresivamente favorecido).

Primary bonus. Cuando el partido que se somete a primarias toma una considerable ventaja sobre aquellos que utilizan otros MCS, resultando premiados por la ciudadanía por haber escogido este método de nominación de candidaturas.

Legitimidad Doble legitimidad. Elección de un candidato que logre la difícil combinación entre legitimidad partidaria y legitimidad popular; es decir, que esté asociado con la ideología o proyecto partidario y que sea capaz de captar más votos, vencer a sus oponentes y obtener una mejor performance electoral.

Retención coalicional. Cada partido ha de retener, en la elección general, a los votantes de las listas internas derrotadas en las primarias.

Participación y Voto sincero: que no se produzca crossing over (trasvasamiento de votantes ajenos) o empoderamiento entrismo (estrategias entristas de electores de otros partidos).

ciudadanos Voto direccionado: que el elector sufrague contemplando su ordenamiento de preferencias real, sin manipular su voto. Y que respete la dirección de su identificación. Intensidad en las preferencias: que el elector tome la decisión de ir a votar en esta instancia por una opción específica con la cual se identifica (con el objeto de posicionar mejor a la fuerza política que más lo representa, votando a su candidato preferido) y no por descarte o por considerarlo un "mal menor".

Fuente: Elaboración propia sobre la base de Key (1955); Epstein (1980); Hirczy (1994); Ansolabehere et al. (1999); Boix (1998); Atkeson (1998); Pierce (1999); Colomer (2002); Rahat y Hazan (2001); Carey y Polga-Hecimovich (2006); Siavelis y Morgenstern (2008); Anduiza Perea y Bosch (2004); Lyne (2005); De Luca et al. (2008); Navia y Rojas Morales (2008); Adams y Merrill III (2008); Kemahlioglu et al. (2009); Serra (2011); Altman (2012); Freidenberg (2016); Piñeiro (2014); Piñeiro (2014); Malamud (2015); Zelaznik (2015); Tullio (2015); Cantillana Peña et al. (2015); Reynoso (2015); Agranov (2011); Navia (2017). 
Tabla 3: Variables, dimensiones e indicadores

\begin{tabular}{|c|c|c|}
\hline Variable & Dimensión & Indicador y valores \\
\hline \multirow{2}{*}{$\begin{array}{l}\text { 1. Reducción y } \\
\text { ordenamiento }\end{array}$} & \multirow[t]{2}{*}{ Reducción de oferta } & $\begin{array}{l}\text { NEP electoral1 (se lo comparará con el NEP promedio des- } \\
\text { de la democratización) }\end{array}$ \\
\hline & & $\begin{array}{l}\text { Cantidad de partidos que no alcanzaron el piso y su peso } \\
\text { en el conjunto del electorado }\end{array}$ \\
\hline \multirow{5}{*}{ 2. Democratización } & $\begin{array}{l}\text { Criterios de ordena- } \\
\text { miento }\end{array}$ & De largo plazo o de corto plazo \\
\hline & MSC anterior & Democráticos (D) o no democrático (ND) \\
\hline & Competencia & $\begin{array}{l}\text { Nro. de listas internas (siendo competida si se presentan al } \\
\text { menos dos por partido) }\end{array}$ \\
\hline & Competitividad & $\begin{array}{l}\text { Ventaja de la lista ganadora (la diferencia entre el } 1^{\circ} \text { y el } \\
2^{\circ} \text { ) }\end{array}$ \\
\hline & Conflictividad & $\begin{array}{l}\text { Alta, media o baja (en función de lo reportado por medios } \\
\text { y analistas) }\end{array}$ \\
\hline \multirow[t]{2}{*}{ 3. Viabilidad } & Bonus & $\begin{array}{l}\text { Resultado obtenido por parte de aquellos partidos que pre- } \\
\text { sentaron primarias competitivas }\end{array}$ \\
\hline & Batacazo & $\begin{array}{l}\text { Diferencia entre el resultado de las internas y el de las ge- } \\
\text { nerales }\end{array}$ \\
\hline \multirow{3}{*}{ 4. Legitimidad } & Doble legitimidad & $\begin{array}{l}\text { Legitimidad popular: Candidato consagrado tras competen- } \\
\text { cia interna }\end{array}$ \\
\hline & & $\begin{array}{l}\text { Legitimidad partidaria: Si se eligió a un Party Adherent } \\
\text { (PA) o un Party Insider (PI) }\end{array}$ \\
\hline & Retención & $\begin{array}{l}\text { De los votantes del partido en la elección general, porcen- } \\
\text { taje de los que lo votaron en interna }\end{array}$ \\
\hline \multirow{3}{*}{ 5. Participación } & & Con fugas posteriores o sin fugas posteriores \\
\hline & $\begin{array}{l}\text { Voto direccionado y } \\
\text { sincero }\end{array}$ & $\begin{array}{l}\text { Porcentaje de ciudadanos habilitados que votaron al candi- } \\
\text { dato en la interna y en las elecciones restantes }\end{array}$ \\
\hline & $\begin{array}{l}\text { Intensidad en la } \\
\text { preferencia }\end{array}$ & $\begin{array}{l}\text { Porcentaje de ciudadanos habilitados que votaron al parti- } \\
\text { do en la interna }\end{array}$ \\
\hline
\end{tabular}


Tabla 4: Situación previa (a la incorporación de la ley) en cada uno de los tres países URUGUAY CHILE

\begin{tabular}{|c|c|}
\hline $\begin{array}{l}\text { Situación } \\
\text { previa }\end{array}$ & ARGENTINA \\
\hline $\begin{array}{ll}\text { Sistema } & \text { de } \\
\text { partidos } & \end{array}$ & $\begin{array}{l}\text { Poco institucionalizado y ato- } \\
\text { mizado; Fragmentado y con } \\
\text { volatilidad partidaria; Con si- } \\
\text { metrías e inequidad entre las } \\
\text { fuerzas políticas; Con fuerzas } \\
\text { inconsistentes en lo ideológico } \\
\text { y programático }\end{array}$ \\
\hline
\end{tabular}

Tipo de par- Constituidos como meros setidos los electorales nominativos, sin estructuras ni afiliados y sin capacidad de incidencia territorial ni representación social; Internamente poco democráticos y con un funcionamiento arcaico

Funciones de Cumplimiento deficitario partidos
Estructurado e institucionalizado; Dos bloques o fami- lias partidarias (el bloque desafiante $\mathrm{y}$ el tradicional) a dos pactos sobrerrepresen- tados; Copamiento coalicio- nal; Oferta partidaria excesi- vamente restrictiva (producto del sistema binominal)
Duopolio político, en torno

Sólidos y estables, aunque internamente fraccionalizados

Estructurados en torno a dos grandes coaliciones; Sólidos y representativos de una cosmovisión ideológica específica candidatos

Vínculos entre dirigentes y partidos Vínculo entre ciu- daria; Importante porción de dadano y los ciudadanos independienpartido

\section{tes que experimenta rechazo} hacia la política y es refractario a alguna/s de las fuerzas políticas existentes

Vínculo entre subsectores

Conflictividad acarreada por la competencia interna; Falta de claridad en las disputas por los cargos partidarios

Criterio para elaborar preferencias ciudadanas
Cumplimiento adecuado

Por convenciones partidarias de base se sustenta crecientemente en estrategias personalistas

Internas abiertas o semi abiertas y selección por cúpulas

Lealtad (salvo excepciones)

Importante porción de la población que milita, adhiere o simpatiza firmemente con alguna de las fuerzas partidarias existentes

Fraccionalización

funcional

Superposición entre los factores partidarios y los ideológi$\cos$
Tensas, pero mayormente leales

Poca adhesión partidaria por parte de la ciudadanía; Bajo nivel de confianza en las instituciones políticas, particularmente en los partidos; Movilización social por fuera de los canales partidarios existentes

Coexistencia de partidos consocios divergentes

Línea demarcatoria ideológica (centrada autoritarismo/democracia) cuyas áreas están representadas por coaliciones
Movilización electoral a nivel
Cuestiones de corto plazo (como el apoyo o no a la gestión vigente, traducido en el eje gobierno-oposición, junto a elementos subjetivos y personales de los candidatos)

Fuente: Elaboración propia sobre la base de González y Vargas (2013); Cantillana Peña et al. (2015); Luna y Rosenblatt (2016); Latinobarómetro (2013); Abal Medina (2009); Jones y Hwang (2007); Ramírez (2015); Tullio (2011); González (1999); Bottinelli (2016a); Moulián (2002); Piñeiro (2014); Zuleta Puceiro (2015). 
Tabla 5: Internas/primarias abiertas en los tres países

\begin{tabular}{|c|c|c|c|}
\hline Características & ARGENTINA & URUGUAY & CHILE \\
\hline $\begin{array}{l}\text { Obligatoriedad para parti- } \\
\text { dos }\end{array}$ & Sí & Sí & No \\
\hline $\begin{array}{l}\text { Obligatoriedad para vo- } \\
\text { tantes }\end{array}$ & Sí & No & No \\
\hline $\begin{array}{l}\text { Carácter vinculante de es- } \\
\text { ta etapa }\end{array}$ & Sí & Sí & Sí \\
\hline $\begin{array}{l}\text { Piso para pasar a la elec- } \\
\text { ción general }\end{array}$ & $\begin{array}{l}1,5 \% \text { de los votos váli- } \\
\text { damente emitidos del } \\
\text { distrito }\end{array}$ & 500 votos & Sin piso \\
\hline $\begin{array}{l}\text { Carácter eliminatorio de } \\
\text { esta etapa }\end{array}$ & Sí & Sí & No \\
\hline ¿Qué categorías se votan? & $\begin{array}{l}\text { Todos los cargos nacio- } \\
\text { nales por separado (y } \\
\text { en las provincias con } \\
\text { PASO) }\end{array}$ & $\begin{array}{l}\text { Solo el postulante que } \\
\text { encabeza el binomio } \\
\text { presidencial }\end{array}$ & $\begin{array}{l}\text { Se ha utilizado para } \\
\text { elecciones presidencia- } \\
\text { les y parlamentarias }\end{array}$ \\
\hline $\begin{array}{l}\text { Congruencia entre entida- } \\
\text { des partidarias nacionales } \\
\text { y subnacionales }\end{array}$ & $\begin{array}{l}\text { Margen de autonomía } \\
\text { para que las provincias } \\
\text { armen alianzas }\end{array}$ & $\begin{array}{l}\text { Las mismas entidades } \\
\text { compiten para cargos } \\
\text { nacionales y locales }\end{array}$ & $\begin{array}{l}\text { Los pactos pueden di- } \\
\text { ferir según se trate de } \\
\text { nivel nacional, parla- } \\
\text { mentario y municipal }\end{array}$ \\
\hline $\begin{array}{l}\text { Situación de perdedores } \\
\text { de primarias }\end{array}$ & $\begin{array}{l}\text { Impedidos de integrar } \\
\text { fórmula }\end{array}$ & $\begin{array}{l}\text { Posibilidad de acuer- } \\
\text { dos e inclusión en la } \\
\text { fórmula presidencial }\end{array}$ & $\begin{array}{l}\text { Pueden presentarse, } \\
\text { pero no para el mismo } \\
\text { cargo y } \\
\text { electoral }\end{array}$ \\
\hline Coaliciones & $\begin{array}{l}\text { Se forjan antes de las } \\
\text { PASO }\end{array}$ & $\begin{array}{l}\text { Preceden largamente } \\
\text { al sistema }\end{array}$ & Preceden al sistema \\
\hline
\end{tabular}

Fuente: Elaboración propia sobre la base de las constituciones nacionales y las normativas electorales de la República Argentina y de la República Oriental del Uruguay y de la República de Chile. 


\section{Uruguay}

Allí, las elecciones internas abiertas se han sucedido desde que fueron adoptadas formalmente en la constitución de 1997. En todas las oportunidades, al menos dos de los partidos principales presentaron competencia (aunque no todas las internas fueron competitivas). En 1999, el Frente Amplio, postuló al líder indiscutido Tabaré Vázquez junto a la candidatura testimonial de Danilo Astori; en el Partido Colorado, Jorge Batlle terminó ganándole a Luis Hierro; en el Partido Nacional, Luis Alberto Lacalle Herrera derrotó a los desafiantes Juan Andrés Ramírez, Alberto Volonté y a otros candidatos secundarios (Buquet y Chasquetti, 2005).

En 2004, en el FA también compitió Vázquez, esta vez como candidato único, pero apoyado por diferentes listas internas. En el Partido Colorado, aún golpeado por las secuelas de la crisis, se presentó Guillermo Stirling, como principal candidato, junto a otros contendientes menores sin chances. En el PN, Jorge Larrañaga le ganó al por entonces insider Lacalle Herrera (Bottinelli, 2016a).

En 2009, en el FA, José Mujica y Danilo Astori se midieron en unas internas tensas y conflictivas (Cardarello y Yaffé, 2011). En esa instancia, contra la mayoría de los pronósticos, el FA obtuvo menor porcentaje que el PN, y el ganador Mujica resultó menos votado individualmente que Lacalle. Ergo, el $\mathrm{PN}$ y su postulante emergieron públicamente como los grandes triunfadores de la jornada.

En 2014, en el FA, Tabaré Vázquez compitió contra la desafiante Constanza Moreira, quien no peleaba por ganar la candidatura presidencial, sino para expresar una corriente en discrepancia dentro del FA (Bottinelli, 2016b). En el Partido Colorado se postulaba Pedro Bordaberry contra el batllista José Amorín y en el Partido Nacional, Luis Lacalle Pou (hijo de Lacalle Herrera) competía contra el candidato favorito y uno de los jefes partidarios Jorge Larrañaga. Dentro del FA y del PC se impusieron los candidatos esperados, Vázquez y Bordaberry, respectivamente. En el PN, en cambio, Lacalle Pou derrotó a Larrañaga, constituyéndose así en el factor sorpresa de la campaña, lo cual aglutinaría a la oposición tradicional (Bottinelli, 2016b).

En la primera vuelta de octubre de 2014, contrariando las expectativas previstas, Vázquez obtuvo una gran diferencia sobre Lacalle Pou (quien no se benefició del batacazo interno obtenido) que derivaría en un escenario casi cantado para el balotaje de noviembre . Finalmente, como era previsible, en la segunda vuelta se impuso Tabaré Vázquez $(56,62 \%$ a $43,38 \%)$.

\section{Argentina}

En el primer proceso eleccionario en el que se aplicaron las PASO (2011) se presentó la mandataria Cristina Kirchner, quien competía como candidata única del FPV, cuyo triunfo final aparecía como cuasi asegurado. Ante esa situación, para evitar profundizar su fragmentación, los partidos opositores también presentaron listas unitarias (Escolar, 2011); con lo cual no hubo competencia en ninguna de las fuerzas políticas que entraron en carrera en esa elección. El oficialismo se impuso tanto en las PASO $(50,24 \%)$ como en las generales $(54,11 \%)$.

Las PASO se volvieron a implementar para los comicios legislativos de medio término de 2013 y luego para las elecciones presidenciales de 2015. En este último proceso eleccionario, en el oficialismo (FPV), se postuló como único candidato, Daniel Scioli - quien no estaba claramente identificado con el kirchnerismo-- Las principales coaliciones de la oposición, forjadas para poder competir en las PASO, fueron: Cambiemos, que llevaba al por entonces jefe de gobierno de la Ciudad de Buenos Aires, Mauricio Macri (PRO), y UNA, que postulaba al ex funcionario kirchnerista Sergio Massa (FR). Los dos contendientes opositores constituyeron sendas coaliciones ad hoc, personalistas y heterogéneas y presentaron una competencia interna testimonial. En las PASO, Scioli (FPV) salió primero $(38,67 \%)$ y Macri (Cambiemos) obtuvo el segundo lugar $(30,11 \%)$. En la elección general, se auguraba una amplia diferencia del primero sobre el segundo, pero sorpresivamente, los dos contendientes estuvieron mucho más cerca de lo previsto $(37,08 \%$ y $34,15 \%$, respectivamente). Finalmente, en el balotaje, triunfó Macri, haciéndose acreedor de un amplio y (programática e ideológicamente) difuso consenso opositor al kirchnerismo (Autor, 2016).

\section{Chile}

En Chile, luego de la incorporación de la nueva ley, en 2013 por primera vez se celebraron elecciones primarias oficiales, organizadas por el Servicio Electoral, las cuales fueron utilizadas por las dos coaliciones principales. En la primaria de Nueva Mayoría, se impuso Michelle Bachelet (PS-PPD-MASPCCH-IC) sobre José A. Gómez (PRSD), Claudio Orrego (PDC) y Andrés Velasco (independiente). En la interna de la Alianza compitieron Andrés Allmand (RN) y Pablo Longueira (UDI), quien reemplazó a su copartidario Laurence Golborne exministro de minería, famoso por liderar el rescate de los 33 mineros- quien fue retirado de la carrera, en medio de un escándalo de corrupción (Castiglio- 
ni, 2014). Longueira triunfó en su interna, pero posteriormente renunció y fue reemplazado por Evelyn Matthei, también de UDI. En la primera vuelta, la candidata de Nueva Mayoría sacó un 46,70\% frente al 25,03\% de su par aliancista. Y en el balotaje la diferencia entre ambas fue de $62,17 \%$ a $37,83 \%$ a favor de Bachelet.

En 2017, la alianza progresista gobernante resolvió no presentarse en las primarias, al no acordar un candidato común en esta instancia. De este modo, dos postulantes compitieron separadamente en las elecciones generales de noviembre: Alejandro Guillier quien, a pesar de simpatizar con el PRSD, se presentó como candidato independiente por la NM y Carolina Goic, por la DC; lo cual, no obstante, le supuso la profundización de la grave crisis interna por la que esta coalición estaba atravesando. En las primarias sólo participaron la alianza derechista Chile Vamos y el Frente Amplio, la fuerza más reciente, surgida ese mismo año, heredera del movimiento estudiantil de 2011, formada por partidos de centroizquierda que buscaban romper con el bipartidismo chileno. Por la coalición de derecha compitieron el ex Presidente Sebastián Piñera (RN), quien lideraba las encuestas, Manuel Ossandón (independiente) y Felipe Kast (Evópoli). La UDI, uno de los principales partidos de la derecha no postuló un representante propio, sino que dio su apoyo al ex mandatario. Finalmente, Piñera triunfó con el $58,4 \%$ de los votos, Ossandón obtuvo 26,2 \% y Kast conquistó un 15,4\% de las voluntades de la derecha. En el FA se enfrentaron los independientes Beatriz Sánchez y Alberto Mayol, resultando ganadora la primera, tal como se esperaba, con el $67,8 \%$ de los sufragios. En suma, en estas internas no tuvo presencia la coalición gobernante y los sectores que participaron en ellas representaban -tanto a nivel popular como institucional- tan solo el $15 \%$ de las preferencias. Por lo tanto, han constituido un tipo de primarias imperfectas, distorsionando el sentido originario de esta etapa como una instancia preliminar con un status semejante al de la elección general.

\section{Análisis de las variables}

Veamos ahora estos elementos en mayor detalle, para poder determinar si la aplicación de este sistema ha tendido a contribuir o no al buen funcionamiento partidario.

\section{Reducción y ordenamiento de la ofer- ta partidaria}

En cuanto a la reducción numérica de la oferta partidaria, como se puede observar, en ninguno de los tres países se logró el objetivo en los términos estipulados previamente. En Uruguay, el NEP electoral se fue reduciendo de elección en elección (no obstante lo cual, en este país no se partía de una cifra problemática); al tiempo que se incrementó el número de contendientes totales (habiéndose postulado, hasta ahora, entre 16 y 20 precandidatos en la instancia interna, cuando la cantidad de candidatos presidenciales promedio entre 1984-1999 era de 14). En la Argentina, en la primera elección se redujo el NEP y además el número de precandidatos en las PASO fue inferior al promedio histórico de postulantes presidenciales; empero, en los comicios presidenciales siguientes ambos valores se incrementaron. En la medida en que con este sistema se desdoblan las instancias electorales, lo que se redujo en cantidad de candidatos postulados, se aumentó en número de precandidatos compitiendo, malogrando el objetivo reductor. Por lo tanto, si se considera al ciclo eleccionario como un todo, debe concluirse que la tendencia ha sido el aumento y no la reducción de los participantes. En los dos países en los que existe un umbral de exclusión (Argentina y Uruguay), la eliminación de competidores tras las internas no resultó significativa (ni en términos numéricos ni de peso electoral de las fuerzas excluidas); ergo, no tuvo efectos sobre el ordenamiento del sistema partidario, como se pretendía originariamente. En Argentina, el elevado piso forzó a las fuerzas pequeñas a coaligarse de antemano y en Uruguay, por el contrario, la existencia de un piso tan bajo no produjo ninguna alteración en el sistema.

En Chile, en ninguna de las dos elecciones hubo una proliferación de precandidatos para la primera fase (en tanto que solo dos coaliciones compitieron en ellas). De todos modos, cabe señalar que en las primeras primarias celebradas (2013), el NEP presidencial se incrementó, como así también el número de candidatos finales que se postularon (9 en total), marcando un record en toda la historia de Chile, que en 2017, apenas se reduciría a 8 contendientes definitivos. A su vez, como allí los partidos pueden elegir postularse a las primarias o no hacerlo (Navia, 2017), esta fase no opera como rueda eliminatoria. Igualmente, la mitad de los partidos que se presentaron en la etapa final no solo no eran competitivos, sino que desde el punto de vista sartoriano, ni siquiera contaban como tales; con lo cual entendemos que a partir del cambio de la legislación, la oferta partidaria se tornó más confusa, sin por ello, haberse vuelto necesariamente más plural 
ni representativa.

En segundo lugar, respecto del criterio en función del cual se produce la ordenación de la oferta partidaria, en Uruguay, suelen prevalecer, efectivamente variables de largo plazo, en Chile también, aunque matizado en función de las alianzas y sus componentes, y en Argentina, claramente, se han impuesto los criterios de corto plazo.

En efecto, en Uruguay, el sufragio es muy partidizado y la ciudadanía vota muy firmemente a los partidos, los cuales son sólidos y estables (Bottinelli, 2016a), al tiempo que éstos se organizan en función de dos bloques discernibles en lo ideológico y en su alineación (o no) con el gobierno (Buquet y Piñeiro, 2011). En Argentina, la existencia de fuerzas que no aglutinaban a sus miembros a partir de algún elemento de afinidad política definido y el cortoplacismo en la configuración de preferencias ciudadanas (Abal Medina, 2009) se han visto reforzados por el carácter transitorio que la ley le adjudica a la alianza/coalición (Art. $6^{\underline{0}}$ ). Así, en la medida en que la coalición se sustenta sobre un arreglo circunstancial, oportunista y pasajero, se dificulta el establecimiento de un criterio relevante y sustantivo (partidario, ideológico, programático, etc.) conforme al cual efectuarse la racionalización y ordenación del sistema partidario. En Chile, pese a que la propensión participativa ciudadana no es tan alta como en Uruguay, de todos modos, muchos votantes pertenecen a espacios ideológicos definidos. El cambio en el sistema partidario introducido con la emergencia del FA en los últimos años, no ha desmantelado las tradiciones ideológicas con las que se asociaba a los pactos postdictadura. Empero, la fase de las primarias, no ha servido para dar lugar a un nuevo clivaje, con sectores y subsectores definidos, sino que ha constituido una muestra muy parcial y poco significativa de cómo un sector minoritario resuelve sus contiendas y antagonismos internos.

\section{Democratización interna partidaria}

En cuanto a los mecanismos de selección de candidaturas utilizados anteriormente, vimos diversidad entre los casos. En Argentina, la mayoría de los partidos utilizaban mecanismos que conllevaban un proceso de designación (Tullio, 2011); pero, como veremos a continuación, la falta de competencia en la mayoría de las internas impidió que las PASO operaran como un MSC democrático. A diferencia de ello, en Uruguay, los partidos implementaban métodos democráticos de selección de candidatos. En Chile, la coalición gubernamental venía desarrollando la selección de candidatos implementan- do procedimientos electivos con participación de los miembros partidarios, desde hacía más de dos décadas (Navia y Rojas Morales, 2008), que paradójicamente en la última elección decidió no sólo no utilizar este método para definir su candidatura unitaria, sino que deliberadamente rompió la unidad para la elección nacional.

Respecto de la competitividad y competencia, en el caso argentino, desde que rige este sistema, el $75 \%$ de las fuerzas participantes postularon una sola candidatura. En 2011, no hubo competencia en ninguna organización. En 2015, sí la hubo, pero las dos alianzas principales que presentaron internas competidas (Cambiemos y UNA), no eran fuerzas preexistentes que resolvían abrir la selección de candidaturas a la ciudadanía común, sino coaliciones ad hoc, formalizadas para este evento electoral, donde los socios secundarios anexaron sus listas a la del postulante presidencial que se daba por ganador definido, estableciendo una pseudocompetencia interna entre candidaturas altamente dispares. Por lo tanto, con las PASO no hubo un incremento de la democracia interna, en tanto que no se otorgaron más opciones a los seguidores estables de partidos presuntamente poco democráticos ya existentes, sino que se crearon artificialmente estas entidades suprapartidarias, para luego poner a sus componentes internos (los partidos coaligados, recientemente integrados) a resolver sus diferencias y definir sus candidaturas públicamente.

En ese sentido, la legislación vigente en Argentina desalienta la competencia interna; en tanto que exige la presentación de una fórmula electoral completa antes de las PASO (Art. 6, Ley 26.571 RA); en Uruguay, en cambio se permite que los sectores internos derrotados puedan quedar representados en las listas oficiales finales (Art. 8, Ley 17.063 ROU). De este modo, en Argentina terminan siendo los órganos partidarios los que determinan las candidaturas partidarias o coalicionales. En consecuencia, en las primarias el ciudadano sólo escoge entre las alternativas que se le proponen, configuradas por un núcleo partidario reducido, concentrado y minoritario.

En Chile, los partidos pueden elegir no seleccionar candidatos mediante primarias, al elegir no competir en ellas. De hecho, en 2013, solo dos de nueve organizaciones utilizaron este mecanismo y en 2017, dos de ocho fuerzas políticas hicieron lo propio . De todos modos, a juzgar por los efectos suscitados, la conveniencia en la participación en tal instancia anida, no en la búsqueda de seleccionar más democráticamente al representante de la formación política, sino en la necesidad de mantener visibilidad y figuración a lo largo del proceso eleccionario (Fernández, 2017). 
Al respecto de la combinación de estos últimos elementos con la cuestión de la conflictividad, observamos que en Uruguay, de las internas abiertas celebradas hasta ahora en las que hubo competencia y competitividad, tres fueron pacíficas y dos fueron conflictivas (Buquet y Chasquetti, 2005; Bottinelli, 2016a). No obstante, ninguno de los tres partidos que celebraron internas competitivas y pacíficas salió primero en la elección general (aunque en un caso se impuso en el balotaje, tras la reversión del resultado originario).

En Chile, como vimos, tanto en 2013 como en 2017, las primarias de sendas coaliciones transcurrieron de un modo tenso y conflictivo. Si bien no se pueden extraer conclusiones directas y determinantes, lo cierto es que después de 2013, ambos frentes (en particular el gobernante) tuvieron que afrontar múltiples y severas desavenencias entre sus componentes. Y luego del evento interno de 2017, si bien Piñera logró sumar el apoyo de sus adustos contrincantes, no consiguió incrementar posteriormente el nivel de adhesión proyectado, obteniendo en primera vuelta el porcentaje más bajo de un candidato presidencial desde la recuperación democrática.

En efecto, la ausencia de competición interna puede ser ventajosa para los partidos en determinadas circunstancias. En Uruguay, el FA se vio beneficiado en la elección de 2004, que fue la única en la que no presentó competencia interna y la única en la que se impuso directamente en la primera vuelta. En Chile, para los comicios de 2005, Soledad Alvear (PDC) retiró su precandidatura interna (Auth, 2005), en pos de lograr una mayor legitimidad y viabilidad de la candidata electa; lo cual, aparentemente habría dado buenos resultados. En Argentina, la rigidez producida por la inamovilidad de las fórmulas presidenciales, que derivó en la inexistencia de competencia presidencial en la primera elección, paradójicamente, también permitió que se evitaran muchas de las pugnas interfaccionales. Por ejemplo, el FPV en 2011 postuló a la presidenta incumbente, lo que permitió alinear a todos los sectores tras su candidatura, y en 2015, fue ella quien terminó de definir la interna presidencial. Es decir, ese partido -históricamente verticalista e internamente poco democrático- evitó tener primarias conflictivas, al precio de no realizarlas; lo que, paralelamente implicó un contrasentido respecto de las metas pretendidas con este mecanismo de selección, que aquella fuerza había introducido.

\section{Viabilidad}

Con respecto al objetivo de que se produjera un batacazo con las primarias, observamos que en Uru- guay, en 2009, el Partido Nacional dio un batacazo general, que no se repitió luego. En 2014, al igual que 2009, este partido fue el que recibió más votos en la jornada de las primarias (lo cual, de algún modo, era esperable, ya que era el único cuya interna presentaba competencia real), aunque el candidato singularmente más votado resultó ser Tabaré Vázquez . Igualmente, ese año, el PN experimentó un batacazo interno, con la victoria de Lacalle Pou, que no redundó en una buena performance en octubre.

En Argentina, en las elecciones de 2011, hubo un batacazo general, efectuado por el FAP (el cual se disolvió al año siguiente); y en los comicios de 2015, se produjo un batacazo interno en el FIT (una coalición que obtuvo alrededor del $3 \%$ de los sufragios ) .

En Chile, para las primarias de 2013 en la coalición de derecha se produjo un batacazo interno del postulante de la UDI, en tanto que las encuestas previas daban como ganador a Andrés Allamand (de RN) (encuestas CERC, 2013; Castiglioni, 2014). De todos modos, este batacazo interno quedaría opacado frente a la pobre performance general de esta alianza.

Vemos que en los tres países hubo un caso, al menos, de batacazo interno, que en ninguno de ellos se replicó en la elección general. Es decir, hasta ahora los candidatos que han dado un batacazo al interior de su partido no han logrado que ese efecto se trasladara a nivel nacional en la elección siguiente. Mientras que los batacazos generales que se replicaron en la instancia posterior, provinieron de fuerzas emergentes que no presentaron competencia interna. Si bien son pocos los casos analizados para sacar conclusiones al respecto, llega a vislumbrarse una correlación entre ausencia de competencia interna y buen desempeño general de la formación, entendiendo que la presentación unitaria pueda ser bien evaluada por la ciudadanía. A su vez, si los únicos batacazos que se replican son los generales (y en fuerzas sin competencia), las primarias pierden efecto informativo y legitimador, para transformarse directamente en una primera vuelta adelantada.

Con respecto a la noción de bonus, en Uruguay, el único caso en el que el mejor posicionado en las internas triunfó en la instancia final (2004), coincidió con la única situación de ausencia de competitividad en alguno de los partidos principales (y la única vez en la que no hubo que recurrir al balotaje); lo cual indicaría que la ciudadanía no habría considerado al mecanismo de selección empleado por el partido como una variable sustancial para el señalamiento de sus preferencias. 
En Argentina, si bien se sostuvo que las PASO habían beneficiado a las fuerzas que habían presentado competencia interna en ellas (Cambiemos y UNA) (Ernesto y Pomares, 2015; Bosoer, 2017), no existe evidencia para sostener que los votantes hayan apoyado a esos frentes teniendo como objetivo (o como uno de sus objetivos) otorgarles un premio por haber resuelto celebrar primarias. Lo que ocurrió, en realidad, fue que los postulantes que encabezaron sendas alianzas electorales (Cambiemos y UNA) estaban entre aquellos que individualmente recibían mayor apoyo ciudadano (Macri o Massa). Es decir, los electores habrían señalado a esos frentes por estar liderados por candidatos que ellos previamente habían seleccionado y no por considerar que los MSC utilizados eran más democráticos que los implementados por sus congéneres.

En Chile, la idea de que las primarias fueran vinculantes tenía como objeto que la ciudadanía pudiera discernir entre candidatos partidarios nominados por distinto tipo de mecanismos internos, otorgándole una ventaja a aquel/los que hiciera/n uso de aquéllas (Mensaje de la ley Nro. 20.640). En los dos casos sucedidos hasta ahora, solo dos coaliciones utilizaron este mecanismo, entre las cuales hubo una gran disparidad de votos (tanto en la interna como en las elecciones subsiguientes). Esto impide establecer - por lo menos, por ahora- una correlación entre el método de nominación implementado y el grado de elegibilidad del candidato consagrado. Cabe agregar que en el proceso eleccionario de 2013, pese a la contundencia de los resultados interpartidarios (70 a 30 a favor de NM), esto no derivó en que la coalición progresista obtuviera la mayoría requerida para imponerse en primera vuelta. En 2017, si bien NM se habría perjudicado por no competir en las primarias (Fernández, 2017), la decisión de no participar provino de la misma situación crítica vivenciada por el pacto de centroizquierda que lo llevó luego a tener una pobre performance en la elección general y a ser derrotado en el balotaje .

\section{Legitimidad}

En relación a la doble legitimidad otorgada por las primarias, solamente puede hablarse de legitimación popular en los casos en los que los contendientes no sólo hayan competido efectivamente, sino que hayan recibido un gran apoyo popular en las primarias (lo cual sucedió en la minoría de los casos). Para que haya habido legitimación partidaria, debería haberse electo además, a un Party Adherent, pero apoyado y referenciado en el partido (como sólo sucedió con Pepe Mujica en 2004 o Lacalle Pou en 2014, en Uruguay). En los demás casos, no se produjo una situación equivalente. Inclusive, en el caso de Chile Vamos, triunfó el candidato "del aparato", derrotando a dos outsiders, que blandían la bandera de la renovación de la política.

El nivel de retención coalicional difiere en función de que las internas sean obligatorias o voluntarias lo cual, a su vez, tiene que ver con el grado de ajuste y congruencia entre selectorado interno (los electores de las primarias) y electorado general. Aunque en cualquier caso, este aspecto se pone en juego cuando existe competencia interna, ya que el reto consiste en evitar la desmovilización de los adherentes del candidato perdedor y las defecciones hacia los competidores externos (Reynoso, 2015; Malamud, 2015).

No es sencillo evaluar este aspecto cuando hay internas opcionales (de las 18 contiendas competidas consideradas aquí, 16 correspondieron a esa modalidad), en la medida en que las fuerzas partidarias suelen recibir mayor apoyo en los comicios generales que en la elección previa; lo cual podría neutralizar las fugas post-internas. Con primarias obligatorias, sí se puede detectar con mayor precisión la deserción de votantes internos de fase en fase. En la Argentina, las fuerzas que presentaron competencia en los comicios de 2015 mostraron un alto nivel de conservación de apoyos entre las instancias eleccionarias ; no obstante lo cual, eso se explica por el hecho de que se trataba de coaliciones asimétricas (Cambiemos y UNA), cuyas listas secundarias tenían una escasa gravitación electoral. De todos modos, el triunfo final de Macri se debió no tanto a los votantes propios que pudo retener de fase en fase, sino al apoyo último de ciudadanos que no lo habían señalado en ninguna de las ocasiones previas (y que lo habrían vislumbrado como "mal menor" frente al kirchnerismo) (Autor, 2016). Esto último (el gradual incremento del apoyo popular desde las primarias hasta el balotaje) también se observó en los otros dos casos en la última elección, dando cuenta de un aspecto que será indagado a continuación: la ampliación de la brecha entre el voto más duro y genuino (el de las primarias) y el más estratégico y desapegado (de la segunda vuelta).

\section{Participación ciudadana}

En Uruguay y Argentina, el incentivo al voto direccionado y sincero en la interna está dado por la obligatoriedad de la presentación de todas las fuerzas que aspiren a competir en los comicios generales. En Chile, por su parte, la exigencia de que los militantes sólo puedan votar a los candidatos de su coalición y la exclusión de afiliados a otros partidos 
previene el voto cruzado de oposicionistas.

En el primer caso, Vázquez fue el mayor receptor del sufragio sincero las tres veces que compitió . Empero, en el último comicio, recibió escasos votos en la interna, aunque en la elección general y el balotaje obtuvo un alto caudal electoral; lo cual ratifica que sus votantes, pese haber reducido su intensidad, mantuvieron consistencia en la direccionalidad de su identificación partidaria.

En Argentina, en 2011, el escenario cuasi definido anticipadamente quitó margen para jugadas especulativas. En 2015, este tipo de voto no se concentró mayoritariamente en Macri; de hecho, sólo un tercio de sus electores finales lo había señalado en ambas instancias previas (Autor, 2016); lo que implica que la mayor parte de sus votantes consideraba como primera preferencia a alguna otra opción que competía previamente.

En el caso chileno, en las primarias de 2013, participaron los dos frentes mayoritarios del país; en 2017 , en cambio, al no competir una de las coaliciones principales, sus simpatizantes no enrolados formalmente quedaban en disponibilidad para intervenir en primarias ajenas; con lo cual no puede excluirse que haya habido una concurrencia de electores fieles no afiliados -presumiblemente, concertacionistas en su mayoría- en las internas de las coaliciones que participaron en ellas.

En relación al punto relativo a la intensidad en la preferencia elegida, éste también varía en función de que las primarias sean optativas u obligatorias para la ciudadanía ; lo cual se vincula con el aspecto ya mencionado referido a la retención coalicional . Cuando las internas son opcionales, como en Uruguay, si un elector toma la decisión de ir a votar en esa instancia, es porque además de ubicar a su alternativa entre las opciones elegibles (Bottinelli, 2016b), probablemente sienta cierto entusiasmo por ésta; por lo tanto es plausible que el voto interno sea relativamente intenso (aun no siendo elevado numéricamente, en su evaluación global) . En Argentina, en cambio, en las primarias, al ser obligatorias -reposando sobre un principio coercitivo (Anduiza Perea y Bosch, 2004)- los electores se vinculan con las fuerzas partidarias que compiten mediante la misma estructura de incentivos que en los comicios generales. Por ello, allí -donde el electorado tiene una baja ligazón con los partidos- el voto de las primarias puede ser tan poco intenso y carente de entusiasmo como el de la elección general.

Chile es el único país de los tres en el que el voto tanto en las primarias como en la elección general es voluntario (igual que en EEUU). Aquí el perfil del votante interno sería similar al de la elección general (como en Argentina); pero con la diferencia de que en este caso, tanto en una elección como en la otra, participan electores en promedio más intensos y politizados que en su vecino transandino. En las primarias de 2013, los sucesivos problemas vivenciados por la coalición de derecha habrían reducido el interés de los simpatizantes de esta fuerza por la dilucidación interna (Castiglioni, 2014), derivando en una alta abstención en la primaria. En 2017, estando en condiciones diferentes, la alianza de derecha concitó una concurrencia superior que en la anterior (captando casi el doble del voto espontáneo que en los comicios internos precedentes). Paralelamente, el FA, una fuerza que había constituido la novedad en esa instancia, no pudo lograr que la intensidad de la participación originaria se tradujera en identificación concreta con una construcción partidaria organizada . Si bien la decisión de participar en las primarias por parte de estas dos coaliciones fue bien saludada públicamente (en contraste con la resolución de no competir por parte de Nueva Mayoría), ninguna recibió un elevado nivel de apoyo intenso ciudadano en esta instancia

En suma, en las primarias celebradas en estos tres países, ninguno de los candidatos partidarios consagrados (y menos aún los ganadores finales), recibió una porción significativa de voto sincero, direccionado e intenso por parte de la ciudadanía común, a la cual, como vimos, se la buscaba hacer partícipe de este proceso, promoviendo su empoderamiento y politización.

\section{Conclusiones}

En este trabajo se decidió evaluar la utilización de internas/primarias abiertas en Argentina, Chile y Uruguay, tres países del Cono Sur en los que éstas poseen regulación oficial; siendo unas obligatorias para partidos y electores, otras sólo para partidos y otras voluntarias tanto para unos como para otros.

Aquí nos hemos focalizado en el análisis de las bases teóricas sobre las que se sustenta la incorporación formal de este método, habiendo aclarado previamente que no han coincidido exactamente con las motivaciones políticas reales de las fuerzas partidarias que propugnaron la reforma en el sistema electoral.

A partir del análisis aquí efectuado, observamos que es muy difícil compatibilizar los objetivos de (1.) crear un sistema de partidos más coherente y ordenado; (2.) lograr mayor democratización; (3.) mayor viabilidad y (4.) legitimidad de los partidos 
y de los candidatos; (5.) mayor participación ciudadana, en la medida en que no es fácil observar el cumplimiento de todas las condiciones requeridas en una misma interna -es decir, reducción de oferta; criterios de ordenamiento de largo plazo; sustitución de un MSC no democrático; competencia; competitividad; ausencia de conflictividad; obtención de bonus; batacazo; doble legitimidad; retención coalicional; voto direccionado y sincero y manifestación de preferencia intensa.

Como conclusión, consideramos que los argumentos teóricos favorables al mecanismo de primarias abiertas reposan sobre una contradicción que vuelve a este procedimiento innecesario y no conveniente. Es decir, las primarias no son necesarias porque los beneficios perseguidos con su aplicación no han respondido a problemáticas comunes -cuya mejor solución habría de encontrarse con este métodoexistentes en todas las sociedades recipientes; sino que su adopción provino de motivaciones políticas particulares, diferentes en cada caso. Se hubiera podido considerar una necesidad de haberse partido de una situación de déficit en los ítems mencionados. Sin embargo, como pudimos observar, esto no era así ya que no había necesidad de reducir el número de partidos ni en Uruguay ni en Chile; mientras que en Argentina sí lo había, pero se requería un criterio reductor coherente que generara alineamientos de largo plazo. La democratización interna partidaria no era menester para las principales fuerzas partidarias uruguayas, chilenas (y algunas de las argentinas); a lo que debe agregarse que la mera existencia formal del método tampoco garantiza una selección más democrática. La viabilidad y legitimación de los candidatos consagrados tampoco podría ser vista como un aspecto respecto del cual existiera un déficit en los casos vistos, ya que en los tres se había incorporado en las últimas décadas una fórmula de elección presidencial más inclusiva, que perseguía tal finalidad (Freidenberg, 2016). Por último, la cuestión de la de participación política difería considerablemente entre los casos: en Argentina, se combinaba baja participación con escasa adhesión partidaria, en Chile, los niveles de movilización no coincidían con los canales partidarios establecidos, y en Uruguay, no había falencias notorias en ese ítem.

Por otro lado, este instrumento tampoco es conveniente porque, a raíz del incumplimiento de las condiciones arriba señaladas, no se tiende a alcanzar el umbral de rendimiento esperado con su aplicación. Es decir, hubo una propensión no a reducir la oferta partidaria, sino a introducir una fase extra, muchas veces innecesaria; con criterios de ordenamiento que, por lo general, no eran de largo plazo; mantenimiento - particularmente en Argentinade mecanismos de selección no democráticos usa- dos en la fase previa a las primarias; poca competencia; bajísima competitividad; alta conflictividad; correlación espuria entre presentación de competencia en primarias y preferencia ciudadana (es decir, dificultad para establecer existencia de bonus); falta de réplica en casos de batacazos (internos y generales); escasas situaciones de candidatos doblemente legitimados; dificultad para retener a los votantes originarios y/o incrementar el rendimiento electoral coalicional; limitados casos de voto direccionado y sincero y manifestación de preferencia intensa (casi ninguno concentrado en ganadores finales). Sucede que la satisfacción de tales condiciones supondría, ante todo, la existencia de un sistema partidario fuerte, compuesto por entidades aglutinantes (partidos políticos o coaliciones pluripartidistas) coherentes, sólidas e internamente democráticas. Pero en tal situación, es factible que estas entidades cuenten con resortes eficaces para resolver asuntos internos, sin necesidad de apelar a la ciudadanía común para la dilucidación de los mismos.

Reiteramos, no obstante, que este método ha sido incorporado en un número reducido de países dentro del subcontinente latinoamericano, los cuales, a su vez, lo han utilizado en pocas ocasiones hasta el momento. Por ello, se continuará con el análisis aquí iniciado, incorporando elementos novedosos que vayan surgiendo con el devenir de los acontecimientos.

\section{Referencias}

Abal Medina, J. M. (2009). Los partidos pequeños saldrán fortalecidos. Argentina Elections.

Adams, J. y Merrill III, S. (2008). Candidate and party strategies in two-stage elections beginning with a primary. American Journal of Political Science, 52(2):344-359.

Agranov, M. (2011). Flip-flopping, intense primaries and the selection of candidates. American Economic Journal: Microeconomics, 8:61-85.

Alcántara Sáez, M. (2002). Experimentos de democracia interna: las primarias de partidos en América Latina. Helen Kellogg Institute for International Studies.

Altman, D. (2012). Universal party primaries and general election outcomes: The case of uruguay (1999-2009). Parliamentary Affairs, 66(4):834855.

Anduiza Perea, E. y Bosch, A. (2004). Comportamiento político y electoral. Ariel, Barcelona. 
Ansolabehere, S., Snyder Jr, J. M., y Stewart III, C. (1999). The effects of party and preferences on congressional roll-call voting. Mimeo, Massachusetts Institute of Technology.

Astarita, M. (2009). El Proyecto atiende una cuestión central como es la excesiva fragmentación de nuestro sistema partidario.

Atkeson, L. R. (1998). Divisive primaries and general election outcomes: Another look at presidental campaigns. American Journal of Political Science, 42(1):256-271.

Auth, P. (2005). Las primarias en la Concertación. Un camino sin retorno. Agenda Pública, 4(7):1015.

Blanco Valdés, R. L. (1996). Ley de bronce, partidos de hojalata (crisis de los partidos y legitimidad democrática en la frontera del fin de siglo). En El debate sobre la crisis de la representación política, pp. 191-230. Tecnos, Madrid.

Boix, C. (1998). Political parties, growth and equality: Conservative and social democratic economic strategies in the world economy. Cambridge University Press.

Bosoer, F. (2017). Las PASO, suman o restan? El Estadista, p. 5.

Bottinelli, O. (2016a). Algo más complicado que internas.

Bottinelli, O. (2016b). Lo más importante de las elecciones del Frente Amplio es la cantidad de votantes.

Buquet, D. y Chasquetti, D. (2005). Elecciones Uruguay 2004: descifrando el cambio. Revista de ciencia política (Santiago), 25(2):143-152.

Buquet, D. y Piñeiro, R. (2011). Participation and Effects of Primary Elections in Uruguay. En ponencia presentada en la IPSA-ECPR Joint Conference, San Pablo, Sao Paulo.

Cantillana Peña, C., Contreras Aguirre, G., y Morales Quiroga, M. (2015). Elecciones primarias y personalización de la política: El caso de las elecciones locales en Chile 2012. Revista de ciencia política (Santiago), 35(2):273-298.

Cardarello, A. y Yaffé, J. (2011). Crónica de una victoria anunciada: estrategias de campaña y desempeños partidarios en las elecciones presidenciales y parlamentarias de 2009. En Del Cambio a la Continuidad, Ciclo Electoral, pp. 23-43. Fin de Siglo, Montevideo.

Carey, J. M. y Polga-Hecimovich, J. (2006). Primary elections and candidate strength in Latin America. The Journal of Politics, 68(3):530-543.
Caruncho, L. (2014). De la maquinaria partidaria a la industria electoral. Continuidades e incentivos institucionales en la Argentina: del radicalismo al kirchnerismo. Revista SAAP, 8(2):491-519.

Castiglioni, R. (2014). Chile: elecciones, conflictos e incertidumbre. Revista de ciencia política (Santiago), 34(1):79-104.

CERC (2013). Encuestas cerc.

Colomer, J. M. (2002). Las elecciones primarias presidenciales en América Latina y sus consecuencias políticas. En M. Cavarozzi y JA Abal Medina (comps.), El asedio a la política. Los partidos latinoamericanos en la era neoliberal, Homo Sapiens, Rosario.

Colomer, J. M. (2004). Cómo votamos: los sistemas electorales del mundo: pasado, presente y futuro. Número Sirsi) i9788497840293. Gedisa, Barcelona.

Cox, G. W. (2004). La coordinación estratégica de los sistemas electorales del mundo: hacer que los votos cuenten. Gedisa,, Barcelona.

De Luca, M., Jones, M. P., y Tula, M. I. (2008). Revisando las consecuencias políticas De las primarias: Un estudio sobre las elecciones De gobernador en la Argentina. Postdata, (13):81-102.

Duverger, M. (1954). Political parties: Their organization and activity in the modern state. Methuen.

Epstein, L. D. (1980). Political parties in Western democracies. Transaction Publishers.

Ernesto, C. y Pomares, J. (2015). Doña flor y sus dos gobiernos.

Escolar, M. (2011). Discusión sobre la nueva ley electoral.

Fernández, P. (2017). Piñera ganó las primarias, ahora la izquierda chilena debe encarar sus demonios.

Freidenberg, F. (2003). Selección de candidatos y democracia interna en los partidos de América Latina. International IDEA, Institute for Democracy and Electoral Assistance, Lima.

Freidenberg, F. (2016). La reina de las reformas: Las elecciones internas a las candidaturas presidenciales en América Latina.

Gallagher, M. y Marsh, M. (1988). Candidate selection in comparative perspective: The secret garden of politics, volumen 18. Sage Publications Ltd, Londres. 
González, C. y Vargas, I. (2013). Génesis y análisis jurídico de la ley Nro. 20.640, que establece el sistema de elecciones primarias para la nominación de los candidatos a Presidente de la República, parlamentarios y alcaldes.

González, L. E. (1999). Las internas partidarias. Columna publicada en el diario EL PAIS, 7:399.

Hernández Valle, R. (2002). La democracia interna de los partidos políticos. En Partidos políticos: democracia interna y financiamiento de precampañas, número 53, pp. 473-492.

Hirczy, W. (1994). The impact of mandatory voting laws on turnout: A quasi-experimental approach. Electoral Studies, 13(1):64-76.

Jones, M. y Hwang, W. (2007). Jefes provinciales de partido: piedra angular del Congreso argentino. En La dinámica de la democracia: representación, instituciones y ciudadanía en Argentina. Prometeo, Buenos Aires.

Kemahlioglu, O., Weitz-Shapiro, R., y Hirano, S. (2009). Why primaries in Latin American presidential elections? The Journal of Politics, 71(1):339-352.

Key, V. O. (1955). Politics, parties, and pressure groups. Crowell, Nueva York.

Kittilson, M. C. y Scarrow, S. E. (2003). Political parties and the rhetoric and realities of democratization. En Democracy Transformed?: Expanding Political Opportunities in Advanced Industrial Democracies, pp. 59-80. Oxford University Press Oxford.

Latinobarómetro (2013). Latinobarómetro.

Lawson, K. (1994). How Political Parties Work: Perspectives from Within. Praeger Publishers.

Luna, J. P. y Rosenblatt, F. (2016). "El camino es la recompensa" o las dificultades de crear un Frente Amplio en Chile.

Lyne, M. M. (2005). Parties as programmatic agents: a test of institutional theory in Brazil. Party politics, 11(2):193-216.

Malamud, A. (2015). Votando con el enemigo.

Meirowitz, A. (2005). Informational party primaries and strategic ambiguity. Journal of Theoretical Politics, 17(1):107-136.

Moulián, T. (2002). El sistema de partidos en Chile. En El asedio a la política, pp. 241-257. Homo Sapiens, Rosario.
Muñoz, R. (2003). El proceso circular de las internas abiertas, obligatorias y simultáneas. En Crisis de Representación y reforma política. CEPRI, Río Cuarto.

Navia, P. (2017). La campaña presidencial en Chile 2017: ¿Un giro hacia la derecha o un voto anti Bachelet? En Seminario dictado en Pontificia Universidad Católica Argentina, Buenos Aires, 13/06/2017., Buenos Aires.

Navia, P. y Rojas Morales, P. (2008). El efecto de la participación electoral: En las primarias para la elección presidencial. De 1999 en Chile. Postdata, (13):193-222.

Page, M. (2016). Breve historia de las paso.

Pérez-Liñán, A. (2002). Argentina y el nuevo patrón de inestabilidad política en américa latina. Revista SAAP, 1(1):167-185.

Pierce, N. (1999). Elecciones Primarias en Estados Unidos.

Piñeiro, R. (2014). Factores de mediano y largo plazo para el análisis del proceso electoral de 2014 en Uruguay. En Ciclo Monitor Electoral, Konrad Adenauer Stiftung- Universidad Católica del Uruguay.

Rahat, G. y Hazan, R. Y. (2001). Candidate selection methods: an analytical framework. Party politics, 7(3):297-322.

Ramírez, I. (2015). Intervención en $\hat{\mathrm{A}}^{\prime} \hat{\mathrm{A}}^{\prime}$ Panel 4: Las tendencias electorales. Las encuestas sobre intención de voto". En Seminario $\hat{A}^{\prime} \hat{A}$ 'Porque la política importa", El Estadista, Buenos Aires.

Reynoso, D. (2015). La carrera de fondo en el kirchnerismo.

Scarrow, S. E., Webb, P., y Farrell, D. M. (2000). From social integration to electoral contestation. En Parties without partisans: Political change in advanced industrial democracies, pp. 343-361. Oxford University Press Oxford, Oxford.

Serra, G. (2011). Why primaries? The party's tradeoff between policy and valence. Journal of Theoretical Politics, 23(1):21-51.

Siavelis, P. y Morgenstern, S. (2008). Political Recruitment and Candidate Selection in Latin America: A Framework for Analysis. En Pathways to power: political recruitment and candidate selection in Latin America, pp. 3-37. The Pennsylvania State University Press, Pennsylvania.

Ting, M. M., Hirano, S., y Snyder Jr, J. M. (2018). Primary Elections and the Provision of Public Goods. The Journal of Politics, 80(2):647-661. 
Tullio, A. (2011). Discusión sobre la nueva ley electoral. En Jornada de discusión nueva ley electoral, Casa Generación Política Sur, Buenos Aires, Buenos Aires.

Tullio, A. (2015). El voto de las PASO se reconfigurará sólo si la alianza es muy heterogénea.

Vargas Machuca, R. (1998). Las reformas institucionales de los partidos políticos. Su relevancia para la gobernabilidad democrática.

Zelaznik, J. (2015). Resultados y Escenarios después de las PASO. En Intervención en seminario Resultados y Escenarios después de las PASO, Buenos Aires: Universidad Di Tella, Buenos Aires. Universidad Di Tella.

Zuleta Puceiro, E. (2015). Zuleta Puceiro: "Veo una elección polarizada entre Macri y Daniel Scioli". 